\title{
Treatment strategies for ischiofemoral impingement: a systematic review
}

\author{
Naoki Nakano ${ }^{1}$ (D) Haitham Shoman ${ }^{1}\left[\right.$ Vikas Khanduja $^{1} \mathbb{C}$
}

Received: 23 February 2018 / Accepted: 22 October 2018 / Published online: 13 November 2018

(c) The Author(s) 2018

\begin{abstract}
Purpose There has been relatively little information about the treatment for ischiofemoral impingement (IFI) because of its rarity as well as the uncertainty of diagnosis. The aim of this study was to provide the reader with the available treatment strategies and their related outcomes for IFI based on the best available evidence, whilst highlighting classically accepted ways of treatment as well as relatively new surgical and non-surgical techniques.

Methods A systematic review of the literature from Medline, Embase, AMED, Cochrane and Google Scholar was undertaken since inception to December 2017 following the PRISMA guidelines. Clinical outcome studies, prospective/retrospective case series and case reports that described the treatment outcome for IFI were included. Animal or cadaveric studies, trial protocols, diagnostic studies without any description of treatments, technical notes without any results, and review articles were excluded.

Results This systematic review found 17 relevant papers. No comparative studies were included in the final records for qualitative assessment, which means all the studies were case series and case reports. Eight studies (47.1\%) utilised non-surgical treatment including injection and prolotherapy, followed by endoscopic surgery ( 5 studies, $29.4 \%)$ then open surgery (4 studies, 23.5\%). Mean age of the participants was 41 years (11-72 years). The mean follow-up was 8.4 months distributed from 2 weeks to 2.3 years. No complications or adverse effects were found from the systematic review.

Conclusion Several treatment strategies have been reported for IFI, and most of them have good short- to medium-term outcomes with a low rate of complications. However, there are no comparative studies to assess the superiority of one technique over another, thus further research with randomised controlled trials is required in this arena. This study explores the wide variety and categories of different treatments used for IFI to guide physicians and shed light on what can be done for this challenging cohort of patients.
\end{abstract}

Level of evidence III.

Keywords Ischiofemoral impingement · Quadratus femoris · Endoscopy · Extra-articular impingement · Systematic review $\cdot$ Hip

\section{Introduction}

Ischiofemoral impingement (IFI) is an uncommon cause of pain and snapping in the hip, buttock, and groin. The pathology occurs because of a reduction of space between the lesser trochanter (LT) and the lateral border of the ischium,

Vikas Khanduja

vk279@cam.ac.uk

1 Department of Trauma and Orthopaedic Surgery - Young Adult Hip Service, Addenbrooke's-Cambridge University Hospitals NHS Foundation Trust, Hills Road, Box 37, Cambridge CB2 0QQ, UK which leads to entrapment of the quadratus femoris (QF) muscle [37]. IFI was first described by Johnson in 1977 in three patients who had undergone an osteotomy of the hip or a hip replacement previously [18]. However, despite it being described almost 40 years ago, it is still frequently misdiagnosed or neglected because of its rarity, and the absence of specific clinical findings and diagnostic tests $[14,26]$. Recently, several studies on the radiological features of IFI and distance between the ischium and the LT, i.e. ischiofemoral distance, have been published [14, 20, 31]. We have also recently reported on the normal ischiofemoral distance (measured as the smallest distance between the lateral cortex of ischial tuberosity and the medial cortex of the LT) and its 
variations using the computed tomography (CT) data of 298 normal hips and found that the mean ischiofemoral distance was $18.6 \mathrm{~mm}$ in females and $23 \mathrm{~mm}$ in males and that it increased by $1.06 \mathrm{~mm}$ for each $1 \mathrm{~mm}$ of offset and dropped by $0.09 \mathrm{~mm}$ with each year of age [14]. In addition, it was reported that narrowed ischiofemoral distance was associated with abnormal magnetic resonance imaging (MRI) signal intensity in the QF muscle [20, 31]. Furthermore, some studies report that the QF muscle signal changes on MRI or symptoms of IFI could be observed in patients without ischiofemoral distance narrowing (e.g. due to tumour [30] or exostosis $[35,38])$, keeping the pathogenesis of IFI uncertain $[20,28]$.

There is relatively little information available on the best management strategy for patients with IFI. This is mainly because of the uncertainty of diagnosis and the fact that conservative treatment such as physiotherapy or activity modification is undertaken as a first step in the management of most cases with IFI [2, 26]. Surgical treatment is reserved for patients in whom conservative treatment fails. Until recently, excision of the LT with an open approach had been recommended as a normal operative technique for IFI with a narrowed ischiofemoral distance [2], however, with the improvement in arthroscopic techniques and devices, some authors report on the entire LT being accessed and resected endoscopically $[10,19,40]$.

Currently, there is a lack of evidence in the literature that provides hip surgeons with evidence-based recommendations on the management of IFI, and no systematic review has been published in this arena thus far. The aim of this study, therefore, was to provide the reader with the available treatment strategies and their related outcomes for IFI based on the best available evidence, whilst highlighting classically accepted ways of treatment as well as relatively new surgical and non-surgical techniques. The objective of this systematic review would be to look at patients from both genders with no demographic restriction, who had any treatment for IFI to treat and alleviate buttock and posterior hip pain with or without distal neuropathic pain radiation and by including the studies reporting on IFI treatment and this would provide the current treatment strategies in practice.

\section{Materials and methods}

\section{Search strategy}

The PICOS tool was adopted to formulate the research question and modified since no comparators were sought in this study [24]. The study included randomised trials, cohort studies, case controls, and case studies as the study designs of interest. The protocol of this systematic review was developed and has been registered in the International
Prospective Register of Systematic Reviews (PROSPERO 2017 CRD42017084855) [17].

Two reviewers searched the online databases (Medline, Embase, AMED, Cochrane, and Google Scholar) for the literature describing the outcomes of treatments for IFI. The Preferred Reporting Items for Systematic Reviews and Meta-Analyses (PRISMA) guidelines were used for designing this study [25]. Database search was conducted on 31st, December 2017 and retrieved articles from the databases since inception to the search date. The electronic search citation algorithm used was: [ischiofemoral (Title/Abstract) OR ischiofemoral (Title/Abstract)]. The search also included the yet to be printed search results and grey literature. Results were pooled and exported to Mendeley reference manager software (Elsevier, Amsterdam, The Netherlands) and duplicates were removed electronically and manually. The two reviewers independently reviewed all the titles and abstracts. The remaining search results were divided equally between the two reviewers and reviewed in duplicate applying the inclusion and exclusion criteria. Any discrepancies at the title and abstract stage as well as the full-text stage were resolved by consensus between the two reviewers and the third more senior author. This process led to $100 \%$ agreement between the authors.

\section{Study selection (inclusion and exclusion criteria)}

Levels 1, 2, 3, 4, and 5 evidence (according to the Oxford Centre for Evidence-Based Medicine [29]) English language studies were eligible for inclusion in the systematic review. We excluded duplicate subject publications within separate unique studies. Both electronically published articles and print journals were included for this review. Clinical outcome studies, prospective case series, retrospective case series and case reports that described the outcomes of treatments for IFI were included. Procedures regardless of open surgery, endoscopic surgery or non-surgical treatment were included. Studies on animal models and basic science studies (e.g. cadaveric studies) were excluded. Studies describing trial protocols without any results, diagnostic studies without any description of treatments, technical notes without any results, and review articles were also excluded. The detailed inclusion and exclusion criteria are shown in Table 1.

\section{Data extraction and analysis}

Both the reviewers independently extracted the relevant study data from the final pool of included articles and recorded this data on a spreadsheet designed a priori in Microsoft Excel 2013 (Microsoft Corporation, Redmond, Washington, USA). Participant-specific demographics extracted from each study included the number of cases, gender distribution, mean age with range (years), mean 
Table 1 Inclusion and exclusion criteria applied to articles identified in the literature

\section{Inclusion criteria}

1. All levels of evidence

2. Written in the English language

3. Studies on humans

4. Studies reporting the outcome of treatment for ischiofemoral impingement

Exclusion criteria

1. Studies describing trial protocols without any results

2. Animal studies

3. Basic science studies (e.g. cadaveric studies)

4. Diagnostic studies without any description of treatments

5 . Technical notes without any results

6. Reviews, systematic reviews

length of follow-up, physical, clinical or radiological condition before the treatment, treatment strategy used in the study, final outcome, and specific comments (if any). Studyspecific demographics extracted from each study included the level of evidence according to the simplified evidence level table from the Centre for Evidence-Based Medicine, Oxford, country where the study was conducted and the year of publication. Studies were then analysed and assessed using the Joanna Briggs Institute Critical Appraisal Checklist (JBICAC) for case reports and case series [17]. A scoring system was implemented based on the findings from the studies. JBICAC scores the answers to its questions as yes, no, unclear or not applicable. We then allocated numbers to each answer where domains answering with yes gets 2 points, unclear gets 1 and no gets 0 . A scoring of 16 and 20 indicated the maximum points of case report and case series, respectively.

\section{Statistical analyses}

The extracted data were then analysed using Microsoft Excel 2013. Statistical analyses in this study focused on descriptive statistics by calculating the mean values for ages and followup times providing an overview summary statistic of ages and follow-up times.

\section{Results}

The initial search found a total of 381 studies from all the databases. The search process led to $100 \%$ agreement among three authors. Duplicates found were 165 articles and were removed. A total of 216 articles were then identified for title screening. One hundred and thirty-four articles were excluded based on the inclusion criteria leaving 82 articles for abstract screening. Fifty-seven articles were then excluded and 25 were included for full-text review.
Table 2 Demographics of the study

\begin{tabular}{ll}
\hline Parameter & \\
\hline Studies analysed & 17 studies \\
Levels of evidence: 4 & 17 studies $(100 \%)$ \\
$\quad$ Case series & 3 studies $(17.6 \%)$ \\
$\quad$ Case report & 14 studies $(82.4 \%)$ \\
Participants (cases) & \\
Male & $15(35.7 \%)$ \\
Female & $27(64.3 \%)$ \\
Mean follow-up time (range) & 8.4 months \\
& $(2$ weeks- \\
Mean participant age (range) & 2.3 years) \\
Approach of treatment & $41.0(11-72)$ years \\
Non-surgical treatment & \\
Open surgery & 8 studies $(47.1 \%)$ \\
Endoscopic surgery & 4 studies (23.5\%) \\
\hline
\end{tabular}

Eventually, 17 studies met all the inclusion criteria and were eligible for critical appraisal, quality assessment and included in the study. Of the participants, $15(35.7 \%)$ were males and 27 (64.3\%) were females (data availability: $100 \%$ ). Mean age of the participants was 41 years (range 11-72 years). The mean follow-up period was 8.4 months distributed from 2 weeks to 2.3 years after the treatment. Study demographics are shown in Table 2. No complication or adverse event was found from the systematic review. The flow chart of the literature search algorithm is shown in Fig. 1. The oldest study included in this review was published in 2011. All the studies included in this systematic review were level 4 studies, which means there were no comparative studies found. Due to lack of homogeneity in studies, a meta-analysis was deemed unsuitable for this study.

Three major treatment strategies were found from this systematic review. Eight studies (47.1\%) utilised non-surgical treatment including injection and prolotherapy, followed by endoscopic surgery (five studies, 29.4\%) and then open surgery (four studies, $23.5 \%$ ). Data extracted from all the studies are shown in Table 3. The outcomes of quality assessment using JBICAC are shown in Tables 4 and 5.

\section{Non-surgical treatment (eight studies)}

The overall quality of the eight articles was $80 \%$ based on the JBICAC ranging from 68.75 to $100 \%$ [3, 11, 21-23, 37, $39,41]$. Four articles reported using conservative treatment (e.g. activity restriction, intake of non-steroidal anti-inflammatory drugs (NSAIDs) or physiotherapy) [11, 23, 37, 41]. Three articles reported the outcome following ultrasound (US) guided injection [21, 22, 39]. One article mentioned deploying prolotherapy with polydeoxyribonucleotide 
Fig. 1 PRISMA flowchart for results of the literature database search

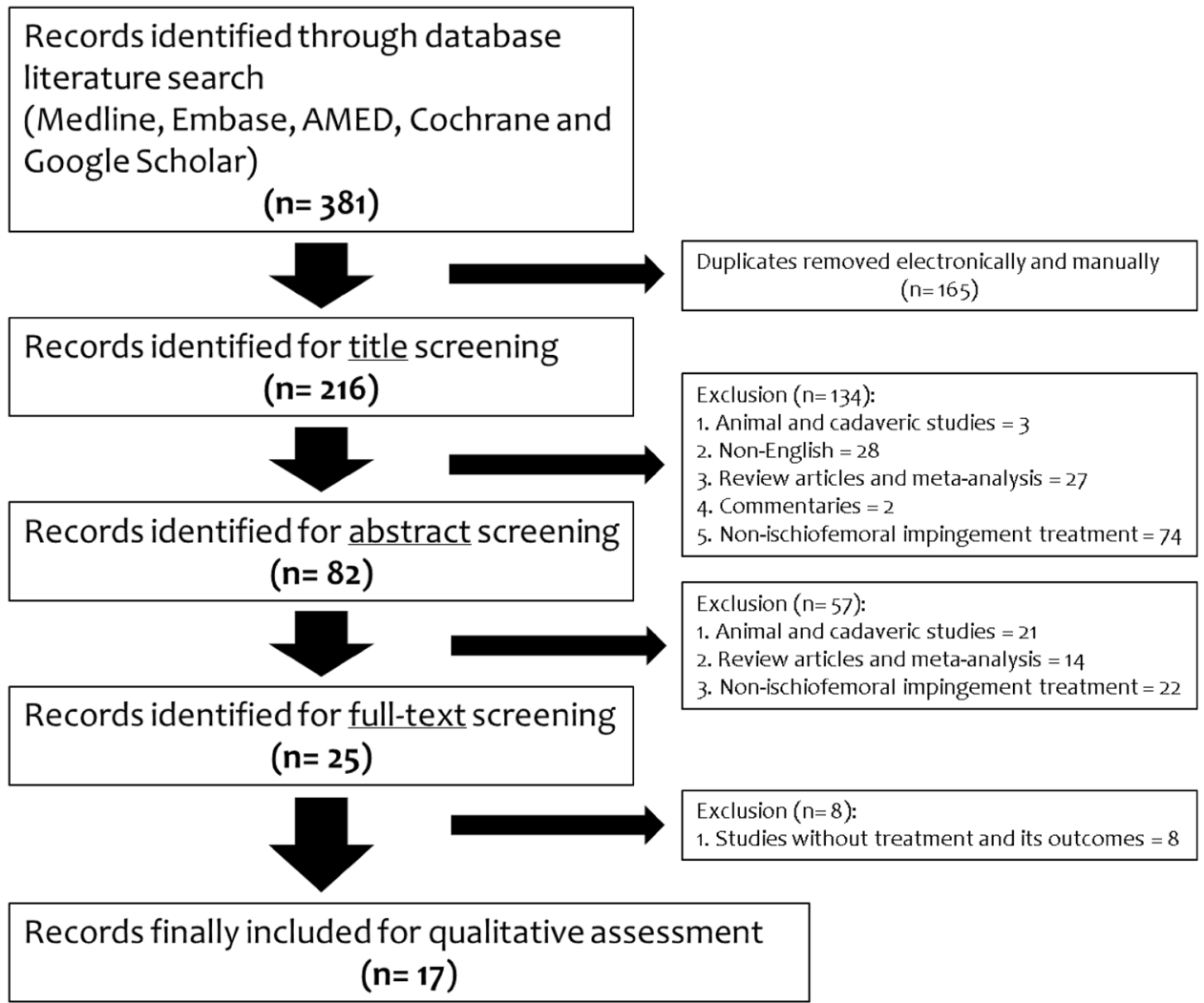

sodium mixed with the local anaesthetics under fluoroscopic and US guidance [3].

\section{Open surgical treatment (four studies)}

The overall quality of studies was $78.1 \%$ based on the JBICAC ranging from 62.5 to $100 \%$. The four open surgical treatment articles were subdivided based on the treatment modality [2, 30, 35, 38]. Two articles reported open surgical resection of the exostosis $[35,38]$. One case report mentioned performing an open surgical resection of the LT [2]. One case report described open surgical resection of a lipomatous tumour in lateral position using a posterior incision [30].

\section{Endoscopic surgical treatment (five studies)}

The overall quality of studies was $91.5 \%$ based on the JBICAC ranging from 81.25 to $100 \%$. All of the five articles were endoscopic surgical resection of the LT in supine position $[10,12,19,33$, 40]. Two articles reported on performing a partial resection of the LT [10, 33] and three articles reported on entire resection of LT $[12,19,40]$.

\section{Discussion}

The most important findings in this study are the availability of three main treatment strategies for IFI being used in current clinical practice. This study reviews all the cases of treatment for IFI (42 cases) with their results reported in the English literature and describes the outcomes of several techniques, which are divided into three categories: nonsurgical treatment, open surgical treatment, and endoscopic surgical treatment. The basic pathology of IFI is that ischiofemoral space is reduced and this leads to compression of the QF muscle within the space causing pain. The QF muscle originates from the external border of the ischial tuberosity and inserts into the upper part of the linea quadrata of the proximal femur and is at risk of compression when the ischiofemoral space is reduced. The aetiology for IFI, i.e. the reason for narrowing of the ischiofemoral space, is variable, which includes ageing (muscle atrophy), female gender (increased width of pelvis), coxa profunda, coxa valga, valgus hip due to proximal femoral osteotomy, Legg-Calve-Perthes disease, total hip replacement with reduced femoral offset or medialized socket, peritrochanteric fractures with involvement of LT, abductor muscle injury causing uncompensated hip adduction during gait, and multiple or isolated exostoses [26]. The treatment in patients with IFI usually starts with conservative approaches such as rest, modification of activities and anti-inflammatory drugs 


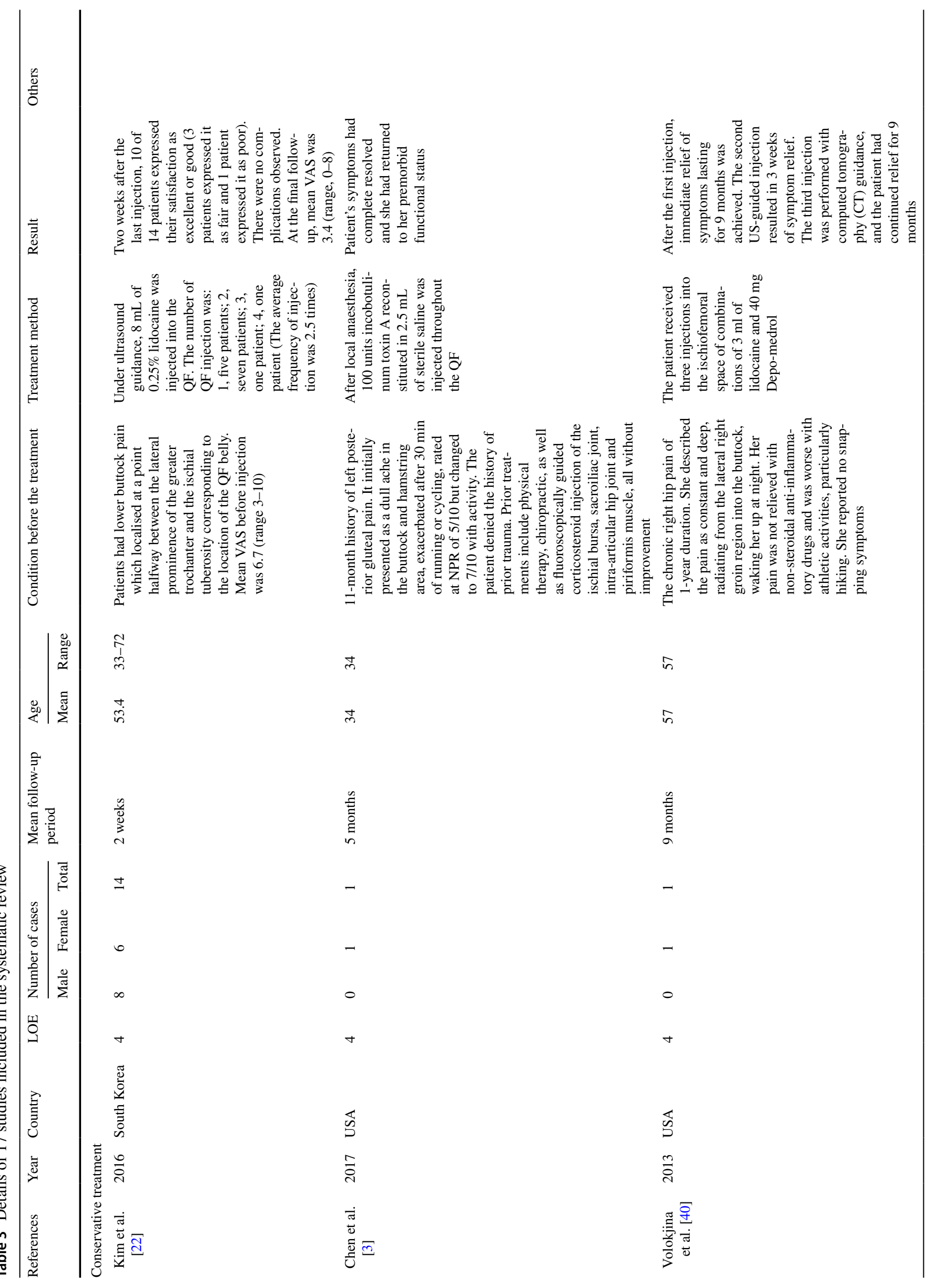




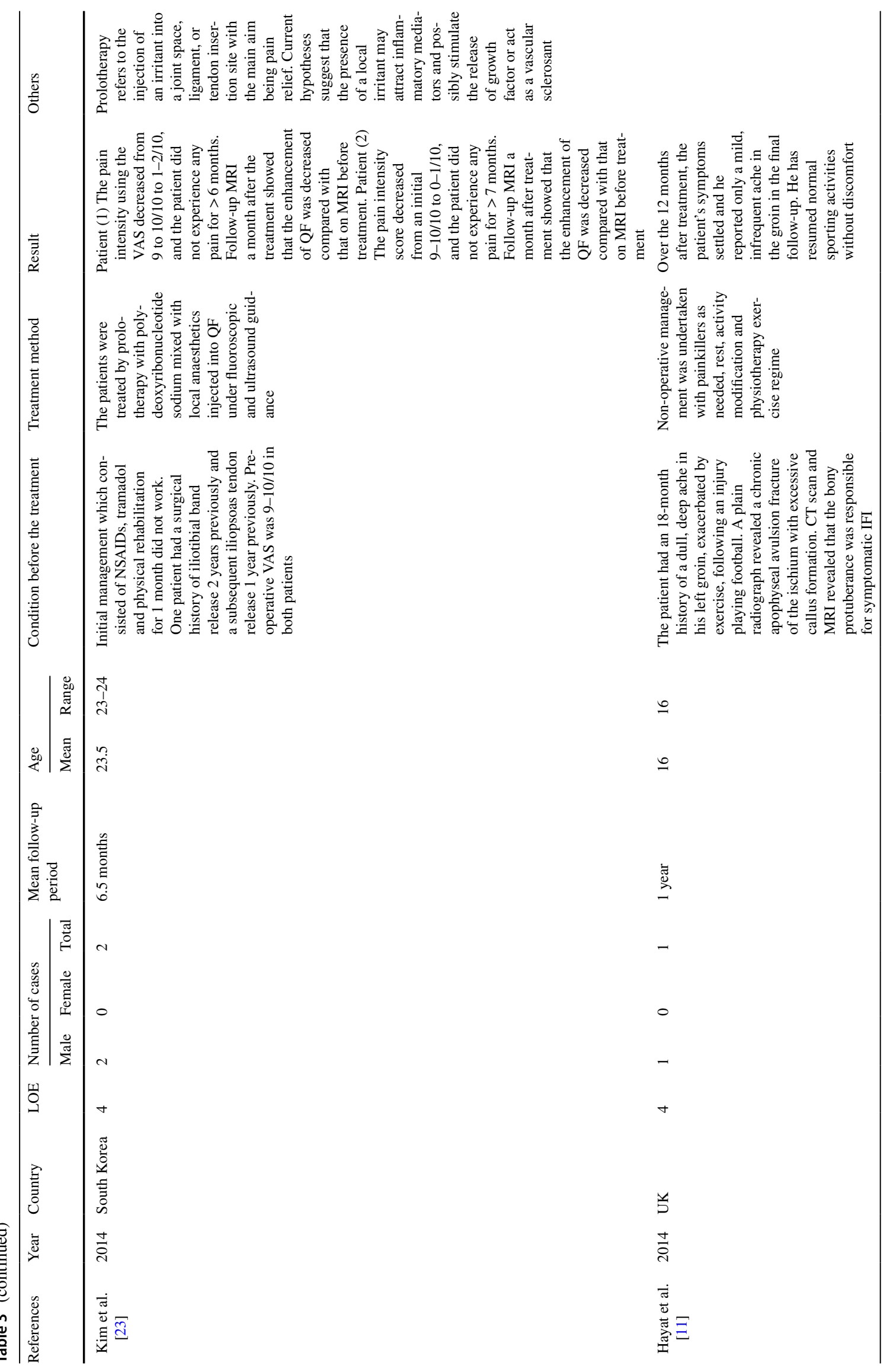




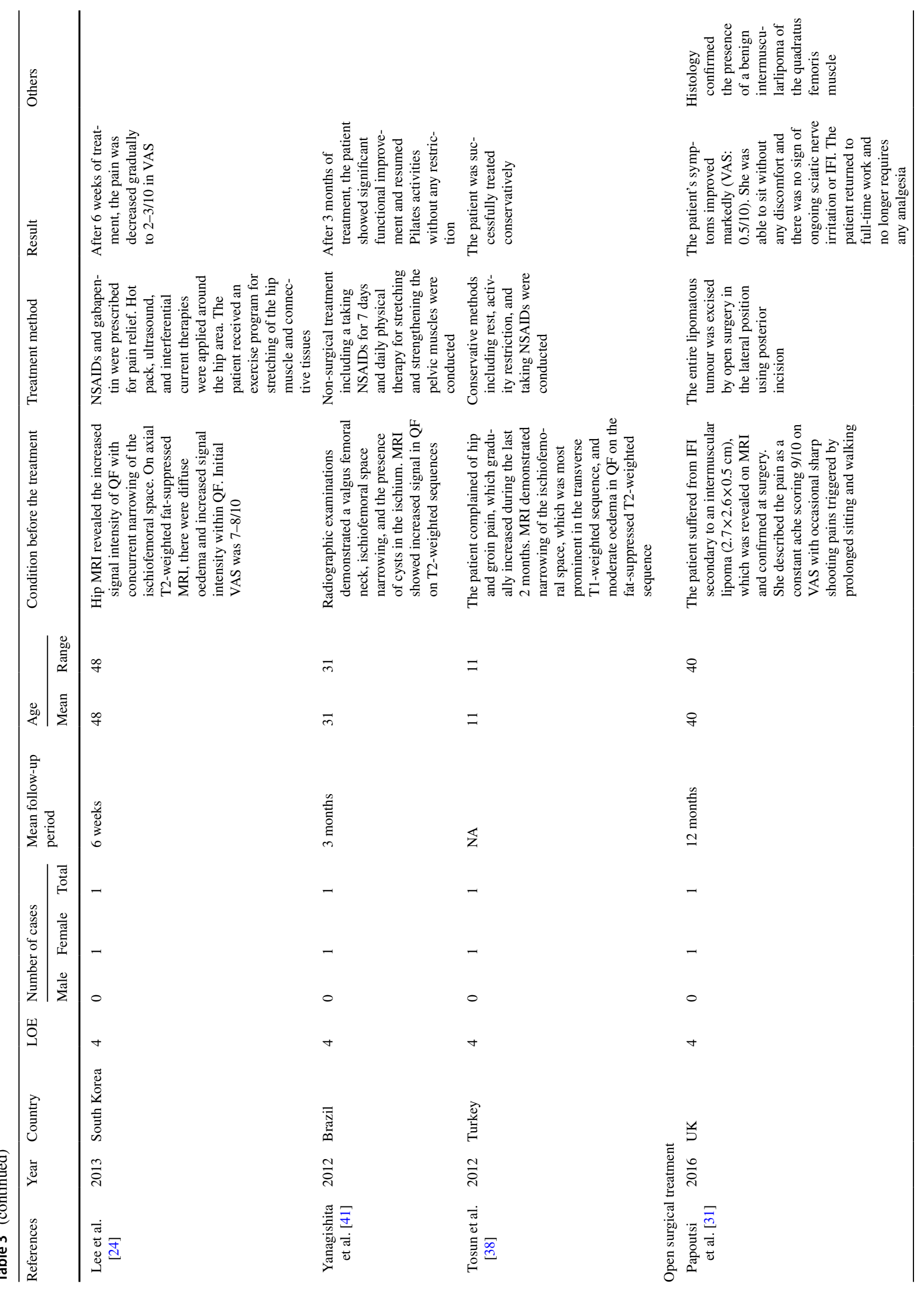




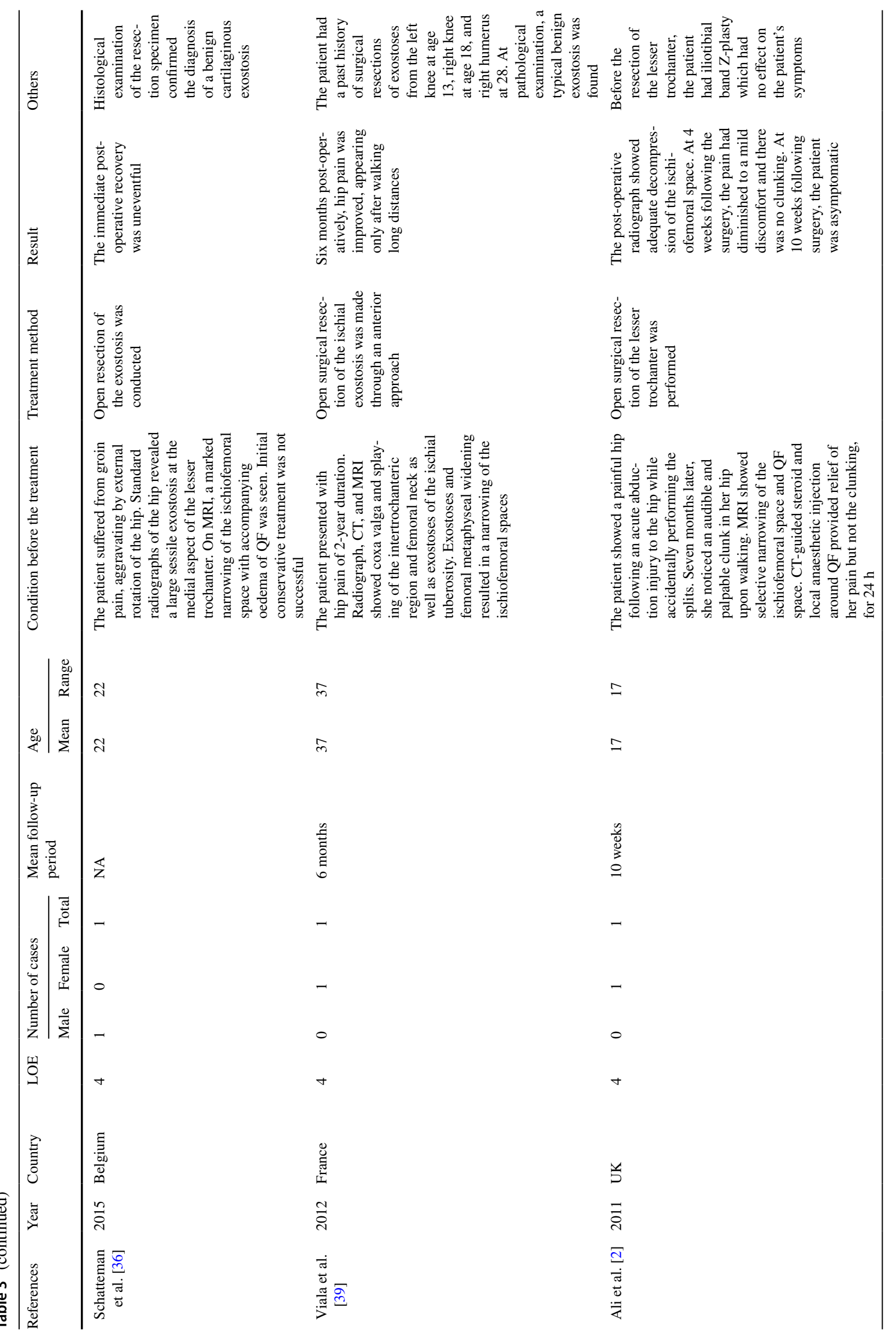




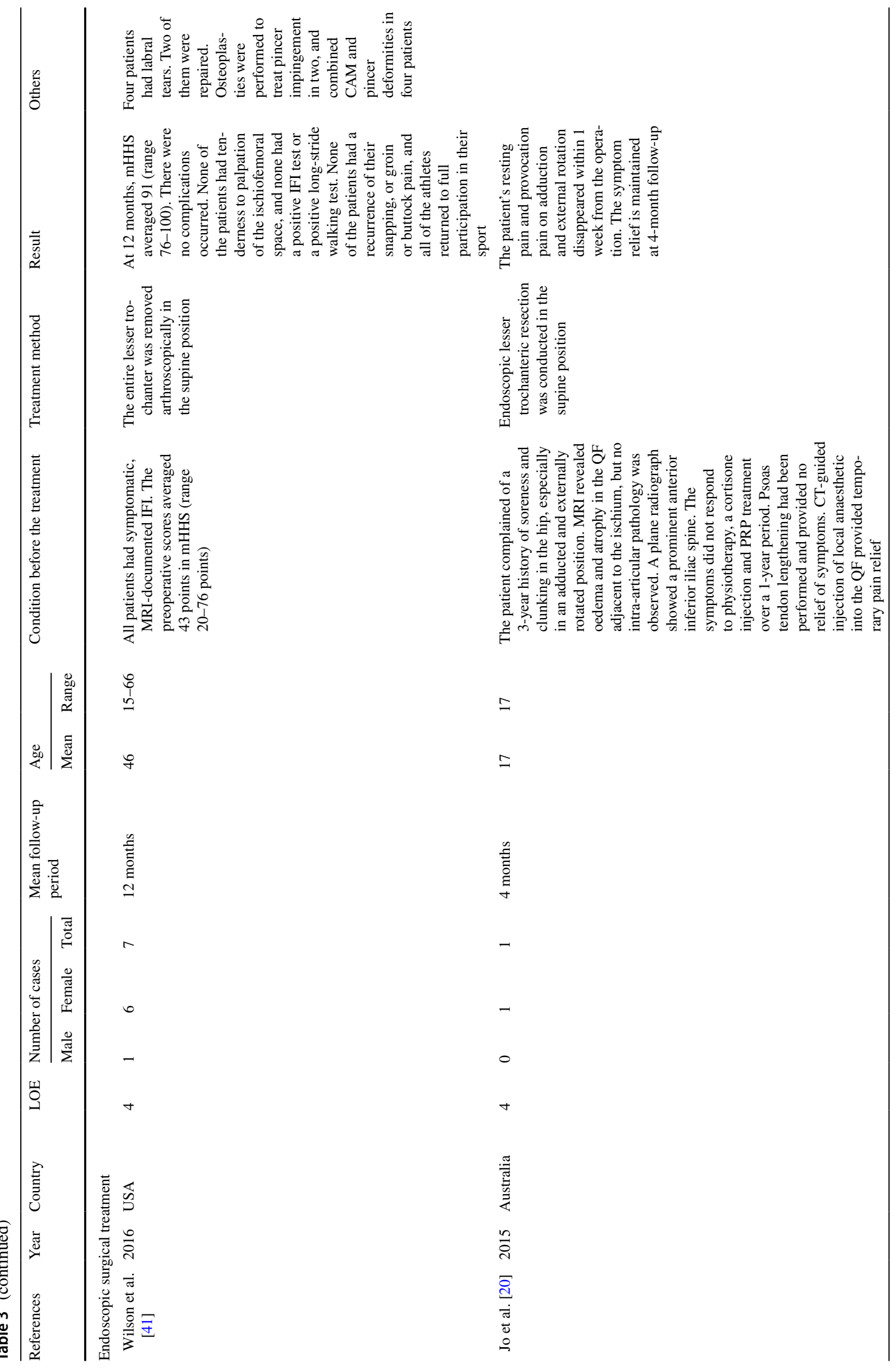




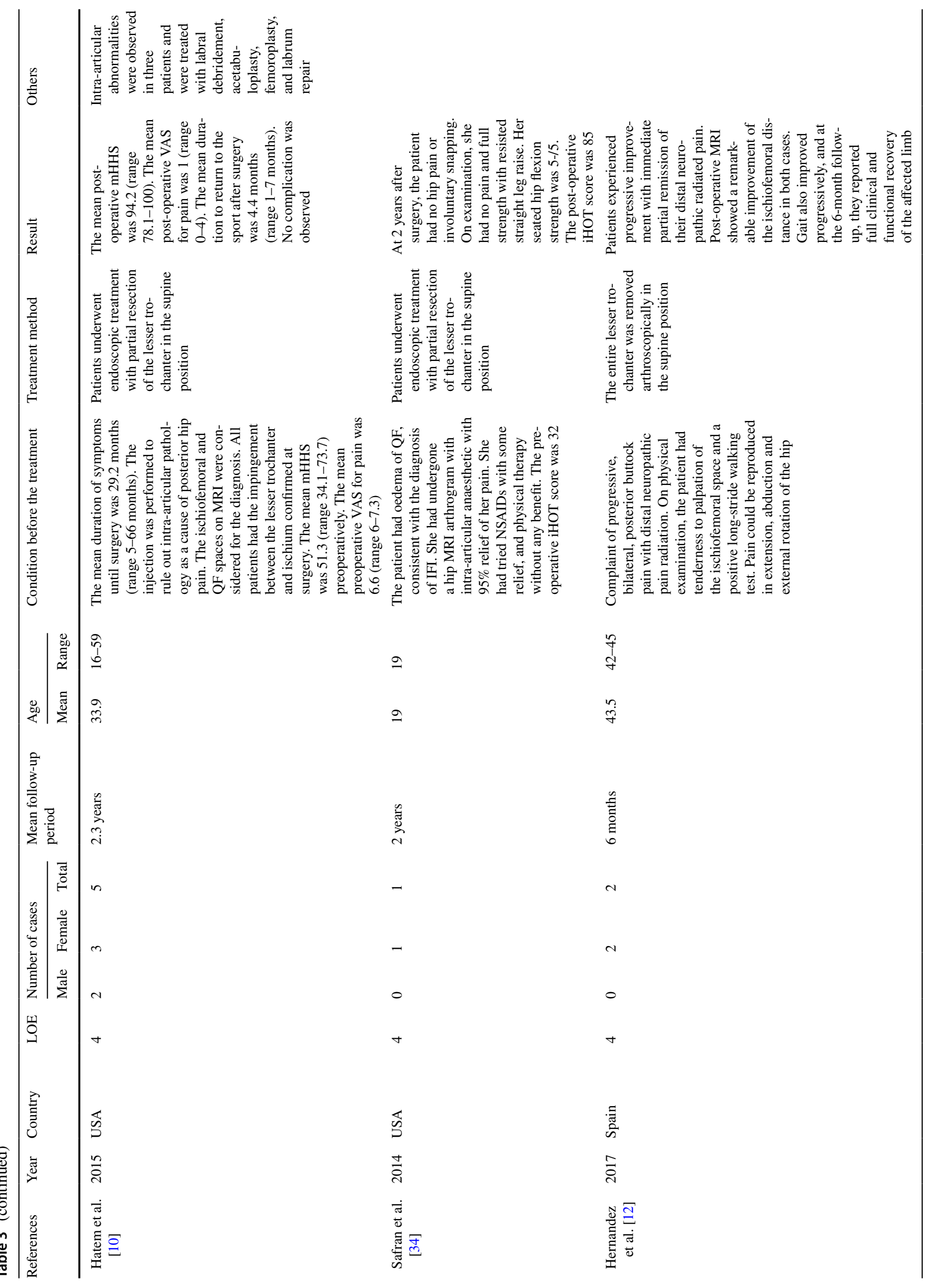


such as NSAIDs. One study [33] mentioned that in their high-volume hip arthroscopy practice, only $5 \%$ of patients diagnosed with IFI required surgical intervention. The objective of this study was to discuss the outcomes of the current treatment strategies for IFI because little has been published on definitive treatment for this condition so far.

\section{Non-surgical treatment}

Of the 17 studies found in the systematic review, five studies reported on conservative treatment $[3,11,23,37,41]$. The studies described the results of 'standard' conservative methods, e.g. the combination of rest, activity modification, taking NSAIDs and gabapentin, physiotherapy, hot packs, and ultrasound-guided injections. All the studies reported good short-term results (from 2 weeks to 1 year) without any complications. This seems to be similar to the management of other impingement syndromes wherein the first line of therapy is usually conservative, because of its less invasive approach and good patient outcomes [4]. Females also tend to have a higher incidence of IFI than males and this might be due to the anatomy of the female pelvis [18]. Females have a wider and a shallower pelvis with a more prominent LT than in males that could lead to IFI [36].

Ultrasound-guided QF muscle injection was reported to be clinically effective [21]. The anatomical location of the QF and its relation to the sciatic nerve could explain why this intervention could be useful. The sciatic nerve is closely located between the anterior surface of the gluteus maximus and the posterior surface of the QF and therefore any inflammation or spasm of this muscle will lead to irritation of the sciatic nerve. Injection of steroid, in this case, would be effective in terms of relieving the pain [34]. Another study reported that one of the ways to treat buttock pain arising from the piriformis muscle was to inject steroids and local anaesthetic [13]. Another study [21] proposed that injection of QF muscle under ultrasound guidance would be an accurate and safe procedure, as for a piriformis muscle injection, an ultrasound-guided injection was known to be more accurate than a fluoroscopically guided injection in a cadaveric model [6] and the two techniques were reported to have no difference in clinical outcomes [8]. Under ultrasound guidance, they injected $8 \mathrm{~mL}$ of $0.25 \%$ lidocaine into the $\mathrm{QF}$ muscle of 14 patients who had deep tenderness localised to a point halfway between the lateral prominence of the greater trochanter and the ischial tuberosity corresponding to the location of the QF muscle belly, and the mean pain score decreased by $49.3 \%$ in 2 weeks after the injection. They reported narrowing of the ischiofemoral space was not found in 3 of 14 patients, so their samples might include patients with other pathology, e.g. piriformis syndrome or myofascial pain syndrome. 


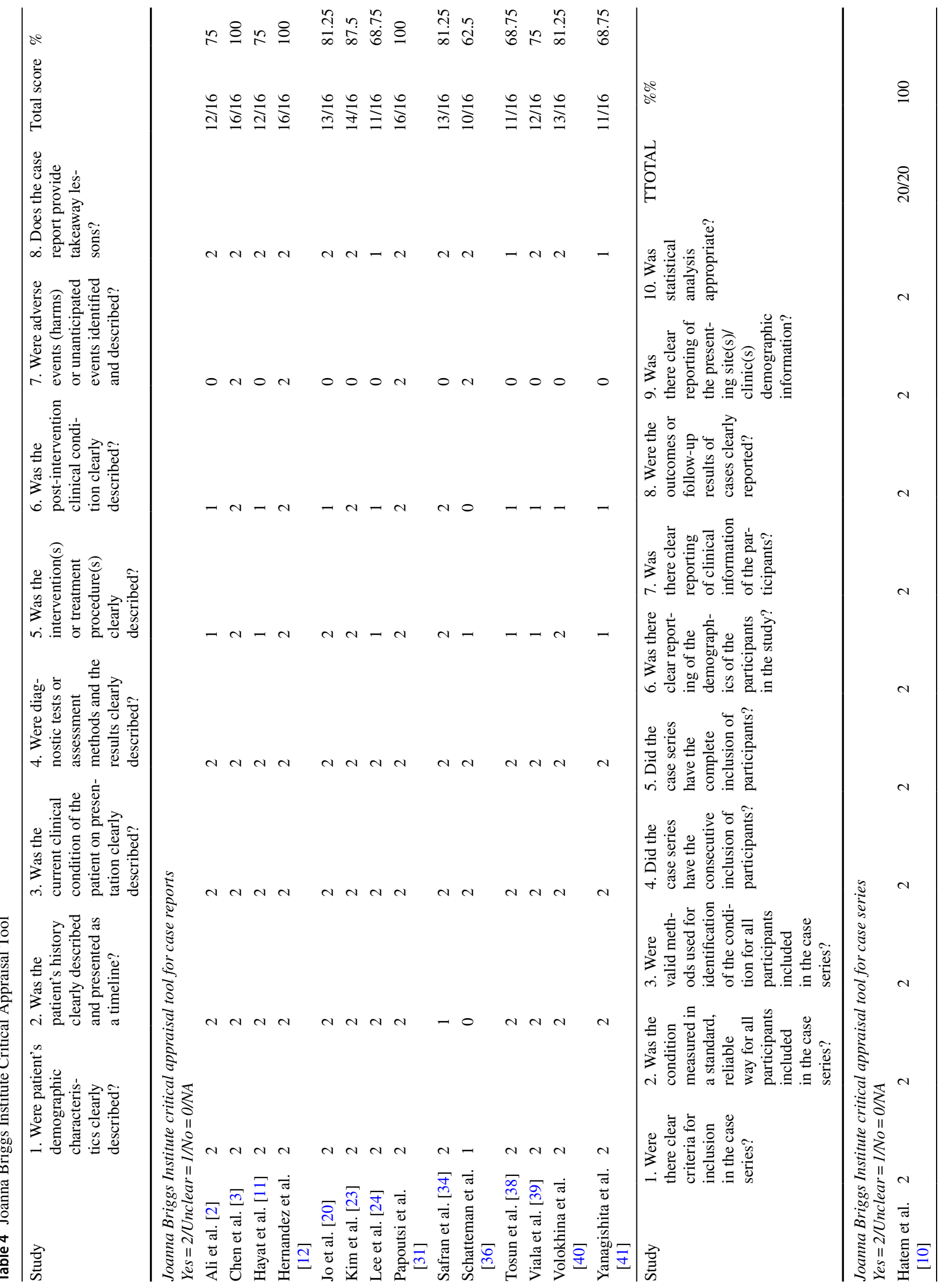




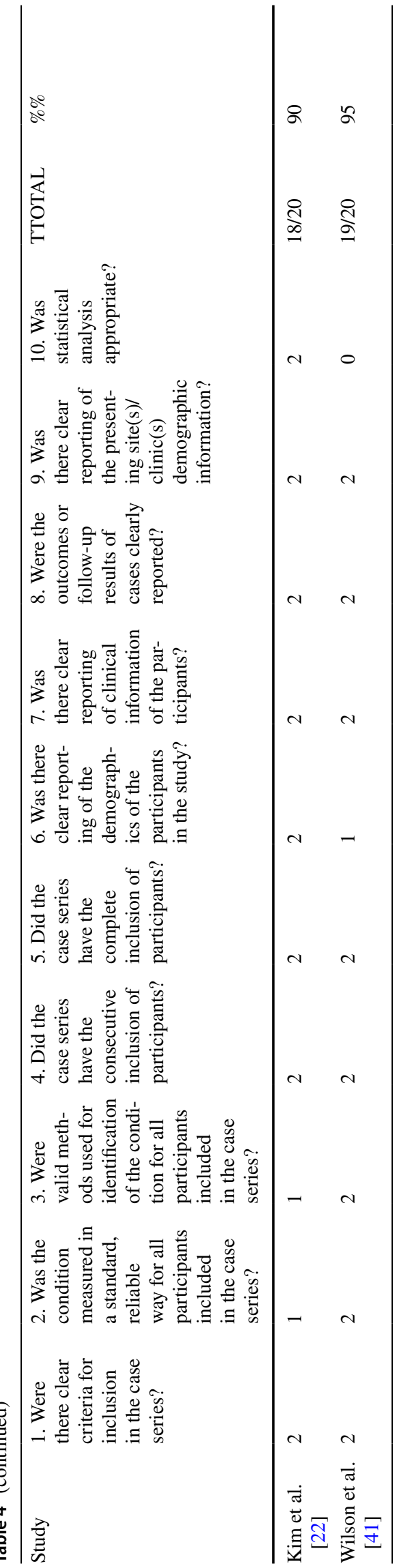

Table 5 Summary of the quality of studies within each major treatment strategy

\begin{tabular}{ll}
\hline Non-surgical treatment & \\
Kim et al. [22] & 90 \\
Chen et al. [3] & 100 \\
Volokjina et al. [40] & 81.3 \\
Kim et al. [23] & 87.5 \\
Hayat et al. [11] & 75 \\
Lee et al. [24] & 68.8 \\
Yanagishita et al. [41] & 68.8 \\
Tosun [38] & 68.8 \\
Mean & 80 \\
Open surgical treatment & \\
Papoutsi [31] & 100 \\
Schatteman [36] & 62.5 \\
Viala [39] & 75 \\
Ali [2] & 75 \\
Mean & 78.1 \\
Endoscopic surgical treatment & \\
Wilson [41] & 95 \\
Jo [20] & 81.3 \\
Hatem [10] & 100 \\
Safran [34] & 81.3 \\
Hernandez [12] & 100 \\
Mean & 91.5 \\
\hline
\end{tabular}

A study [22] reported the outcome of ultrasound-guided prolotherapy with polydeoxyribonucleotide sodium for patients with IFI. Prolotherapy refers to the injection of an irritant into a specific site with the main aim being pain relief, while the mechanism is not completely understood. The presence of a local irritant might attract inflammatory mediators and possibly stimulate the release of growth factors or act as a vascular sclerosant [1, 32]. After prolotherapy, the visual analogue scale (VAS) pain score was found to be minimal (0-1/10), and follow-up MRI revealed a slightly decreased enhancement of the QF muscle compared with that before prolotherapy. They concluded that prolotherapy could be an efficacious treatment option for patients with IFI because the therapeutic effect of steroid injections has only been reported to last from 1 week to 1 month [2] while prolotherapy showed a long-term effect for $>6$ months.

Injections with Botox have also been increasingly used due to its mechanism of action and improvement in patient outcomes. Botox chemodenervation acts by increasing the "space-to-content", which may reduce muscle compression in impingement syndromes [7]. This mechanism of action was reported where Botox was used to treat chronic exertion compartment syndrome where pain faded and function improved [16]. 


\section{Open surgical treatment}

Of the 17 studies found in the systematic review, four studies reported on open surgical treatment for IFI [2, 30, 35, 38]. Two of them reported on the excision of ischial exostosis $[35,38]$, one reported on excision of a lipomatous tumour [30], and another study described the resection of the LT [2]. The pathologic lesion was accessed by either an anterior approach or lateral approach using the trochanteric flip or through splitting of the iliotibial band.

Although no complications related to the open approach were reported, these invasive approaches can potentially endanger the neurovascular structures around the lesion, which can lead to potential delays in rehabilitation. The potential structures in danger are the medial and lateral femoral circumflex arteries, which course on the upper border of the QF muscle [27]. A cadaveric dissection study described that the medial circumflex artery was located on an average of $18 \mathrm{~mm}$ from the LT [9]. A very careful and meticulous approach is therefore mandatory when approaching the superior and posterior portions of the LT to avoid subsequent avascular necrosis of the femoral head. Furthermore, the resection of the LT requires detachment of the iliopsoas tendon [33], which risks persistent weakness of hip flexion.

\section{Endoscopic surgical treatment}

Of the 17 studies in the systematic review, five studies reported on the use of endoscopic surgical management $[10,12,19,33,40]$. All of them reported on partial or entire resection of the LT and good short- to medium-term outcomes (from 4 months to 2.3 years) without any neurological or vascular complication. Although the endoscopic approach is useful for visualisation of the LT and ischiofemoral space, a concern that arises when using this technique is the increased risk of damaging the femoral circumflex artery as well as the perforating femoral artery which could explain why many arthroscopic surgeons have not embarked on utilising this approach [10]. Endoscopic surgery is considered as a minimally invasive surgical decompression technique with fewer complications compared with the open procedure. The psoas tendon has shown some potential for regeneration after its release following endoscopic surgery [15]. Although endoscopic surgery is performed to treat IFI caused by narrowing of the IFI space, it could also help to debride the compromised QF muscle [20].

Due to the location of the LT, the arthroscopic procedure can be approached either anteriorly or posteriorly. A study [10] described the posterior approach and reported favourable outcomes without any complications. However, at the level of the LT, the sciatic nerve is located about $4 \mathrm{~mm}$ from the femoral border [5], thus it can be in danger of injury unless it is approached very carefully. Another study [19] mentioned that the anterior approach was better than posterior approach because the anterior approach can avoid the need to divide the QF muscle and it minimises the risk of damage to the sciatic nerve as well as circumflex femoral vessels.

Partial resection of the LT allowed widening of the ischiofemoral space without releasing all of the iliopsoas tendon insertions, as well as a potentially decreased risk of stress fracture in comparison with complete resection [10]. This fact may be of particular important to high-performance athletes with IFI. A study [33], that reported the entire resection of the LT, partly admitted this risk by mentioning "with this patient accepting the almost assured risk of persistent hip flexor weakness". However, another study [40] insisted that the entire LT should be removed to prevent persistence of the LT impingement due to inadequate resection of bone, which might occur with a partial resection since a thorough dynamic post-resection assessment for impingement cannot be completed with the patient in the supine position on the operating table.

The endoscopic approach seems to have many advantages when compared with the open approach especially in terms of the extent of soft tissue damage. However, care should be taken to remove as much bony debris as possible to reduce the risk of heterotopic ossification [33].

The strengths of this systematic review include the pursuit of knowledge in an important arena that has scarce published information and remains a topical subject for sports physicians and surgeons alike. The methodology is sound and encompasses a broad-based and comprehensive systematic literature search of multiple databases with multiple reviewers allowed for a very inclusive approach to capturing the vast majority of the existing literature. In addition, the included studies were critically appraised using a validated quality measurement tool [17].

Nonetheless, there are limitations which include the inclusion of English only studies and the overall low level of evidence available in the included studies on this topic (level 4 studies only). Non-prospective designs are prone to data inaccuracy as well as missing information, which subjects them to selection and source bias. Publication bias should also be recognised, and these may diminish the accuracy of the data collected and therefore limits the quality of a systematic review without a doubt.

While this current level of evidence reflects the novel and emerging nature of the treatment strategies for IFI, future studies should address comparative effectiveness of the various treatment options in this arena. Most of the studies lacked quantitative metrics in their analysis and hence quantitative conclusions could not be drawn on recommending one treatment strategy over another. To make any specific recommendations for orthopaedic surgeons with regards to treatment decisions, adequately powered long-term 
comparative studies focusing on two or three specific methods of treatment need to be conducted in the future.

\section{Conclusion}

Although there are several different treatment techniques reported, the current best evidence does not support any one treatment technique as a superior method for treating IFI. There, unfortunately, remains a paucity of comparative studies, which makes it difficult to perform a meaningful assessment of the outcome of each procedure. Of the 17 studies found in the systematic review, conservative treatment as well as open/endoscopic surgical resection of the LT, were well-reported, though they were only described in limited case series and case reports.

Author contributions NN, HS and VK contributed to study design, method design, data/statistical analysis, and data interpretation. NN, HS and VK did the literature search and systematic review. NN and HS prepared the initial draft of the article. NN, HS and VK critically revised the article for important intellectual content. VK was responsible for the idea the overall design of the study and is the guarantor.

Funding The senior author has protected research time, which is supported by the Cambridge Arthritis Research Endeavour (CARE).

\section{Compliance with ethical standards}

Conflict of interest No support from any organisation for the submitted work; no financial relationships with any organisations that might have an interest in the submitted work in the previous 3 years; no other relationships or activities that could appear to have influenced the submitted work.

\section{Ethical approval Not required.}

Data sharing No additional data available.

Declaration of transparency The lead author affirms that this manuscript is an honest, accurate, and transparent account of the study being reported; that no important aspects of the study have been omitted; and that any discrepancies from the study as planned (and, if relevant, registered) have been explained.

Open Access This article is distributed under the terms of the Creative Commons Attribution 4.0 International License (http://creativeco mmons.org/licenses/by/4.0/), which permits unrestricted use, distribution, and reproduction in any medium, provided you give appropriate credit to the original author(s) and the source, provide a link to the Creative Commons license, and indicate if changes were made.

\section{References}

1. Alfredson H, Ohberg L (2005) Neovascularisation in chronic painful patellar tendinosis? Promising results after sclerosing neovessels outside the tendon challenge the need for surgery. Knee Surg Sports Traumatol Arthrosc 13(2):74-80
2. Ali AM, Whitwell D, Ostlere SJ (2011) Case report: imaging and surgical treatment of a snapping hip due to ischiofemoral impingement. Skelet Radiol 40(5):653-656

3. Chen YT, Jenkins KM (2018) Ultrasound finding of ischiofemoral impingement syndrome and novel treatment with botulinum toxin chemodenervation: a case report. PM R 10(6):665-670

4. Emara K, Samir W, Motasem EH, Ghafar KAEL (2011) Conservative treatment for mild femoroacetabular impingement. J Orthop Surg 19(1):41-45

5. Ericksen ML, Swenson JD, Pace NL (2002) The anatomic relationship of the sciatic nerve to the lesser trochanter: implications for anterior sciatic nerve block. Anesth Analg 95(4):1071-1074

6. Finnoff JT, Hurdle MFB, Smith J (2008) Accuracy of ultrasoundguided versus fluoroscopically guided contrast-controlled piriformis injections. J Ultrasound Med 27(8):1157-1163

7. Fortuna R, Vaz MA, Youssef AR, Longino D, Herzog W (2011) Changes in contractile properties of muscles receiving repeat injections of botulinum toxin (Botox). J Biomech 44(1):39-44

8. Fowler IM, Tucker AA, Weimerskirch BP, Moran TJ, Mendez RJ (2014) A randomized comparison of the efficacy of 2 techniques for piriformis muscle injection. Reg Anesth Pain Med 39(2):126-132

9. Gautier E, Ganz K, Krügel N, Gill T, Ganz R (2000) Anatomy of the medial femoral circumflex artery and its surgical implications. J Bone Jt Surg Br 82(5):679-683

10. Hatem MA, Palmer IJ, Martin HD (2015) Diagnosis and 2-year outcomes of endoscopic treatment for ischiofemoral impingement. Arthroscopy 31(2):239-246

11. Hayat Z, Konan S, Pollock R (2014) Ischiofemoral impingement resulting from a chronic avulsion injury of the hamstrings. BMJ Case Rep 2014:bcr2014204017

12. Hernandez A, Haddad S, Nuñez JH, Gargallo-Margarit A, Sallent A, Barro V (2017) Ischiofemoral impingement syndrome: outcomes of endoscopic resection of the lesser trochanter. Clin Orthop Surg 9(4):529-533

13. Honorio BT, Katz JA, Benzon HA, Iqbal MS (2003) Piriformis syndrome: anatomic considerations, a new injection technique, and a review of the literature. Anesthesiology 98(6):1442-1448

14. Hujazi I, Jones T, Johal S, Bearcroft P, Muniz-Terra G, Khanduja V (2016) The normal ischiofemoral distance and its variations. J Hip Preserv Surg 3(3):197-202

15. Ilizaliturri VM, Camacho-Galindo J (2010) Endoscopic treatment of snapping hips, iliotibial band, and iliopsoas tendon. Sports Med Arthrosc 18(2):120-127

16. Isner-Horobeti M-E, Dufour SP, Blaes C, Lecocq J (2013) Intramuscular pressure before and after botulinum toxin in chronic exertional compartment syndrome of the leg. Am J Sports Med 41(11):2558-2566

17. Joanna Briggs Institute Critical Appraisal Checklist, JBI-CAT. Critical Appraisal Tools_-JBI. http://joannabriggs.org/research/ critical-appraisal-tools.html. Accessed Nov 2018

18. Johnson KA (1977) Impingement of the lesser trochanter on the ischial ramus after total hip arthroplasty. Report of three cases. J Bone Jt Surg Am 59(2):268-269

19. Jo S, O'Donnell JM (2015) Endoscopic lesser trochanter resection for treatment of ischiofemoral impingement. J Hip Preserv Surg 2(2):184-189

20. Kassarjian A (2008) Signal abnormalities in the quadratus femoris muscle: tear or impingement? Am J Roentgenol 190(6):W379

21. Kim D-H, Yoon DM, Yoon KB (2016) Ultrasound-guided quadratus femoris muscle injection in patients with lower buttock pain: novel ultrasound-guided approach and clinical effectiveness. Pain Phys 19(6):E863-E870

22. Kim W-J, Shin H-Y, Koo G-H, Park H-G, Ha Y-C, Park Y-H (2014) Ultrasound-guided prolotherapy with polydeoxyribonucleotide 
sodium in ischiofemoral impingement syndrome. Pain Pract 14(7):649-655

23. Lee S, Kim I, Lee SM, Lee J (2013) Ischiofemoral impingement syndrome. Ann Rehabil Med 37(1):143-146

24. Methley AM, Campbell S, Chew-Graham C, McNally R, Cheraghi-Sohi S (2014) PICO, PICOS and SPIDER: a comparison study of specificity and sensitivity in three search tools for qualitative systematic reviews. BMC Health Serv Res 14(1):579

25. Moher D, Liberati A, Tetzlaff J, Altman DG (2009) Preferred reporting items for systematic reviews and meta-analyses: the PRISMA statement. PLoS Med 6(7):e1000097

26. Nakano N, Yip G, Khanduja V (2017) Current concepts in the diagnosis and management of extra-articular hip impingement syndromes. Int Orthop 41(7):1321-1328

27. Nakano N, Lisenda L, Khanduja V (2017) Pseudoaneurysm of a branch of the femoral circumflex artery as a complication of revision arthroscopic release of the iliopsoas tendon. SICOT J 3:26

28. O'Brien SD, Bui-Mansfield LT (2007) MRI of quadratus femoris muscle tear: another cause of hip pain. Am J Roentgenol 189(5):1185-1189

29. OCEBM Levels of Evidence-CEBM. http://www.cebm.net/ blog/2016/05/01/ocebm-levels-of-evidence/. Accessed Nov 2018

30. Papoutsi D, Daniels J, Mistry A, Chandraseker C (2016) Ischiofemoral impingement due to a lipoma of the ischiofemoral space. BMJ Case Rep 2016:bcr2015213210

31. Patti JW, Ouellette H, Bredella MA, Torriani M (2008) Impingement of lesser trochanter on ischium as a potential cause for hip pain. Skelet Radiol 37(10):939-941

32. Rabago D, Slattengren A, Zgierska A (2010) Prolotherapy in primary care practice. Prim Care 37(1):65-80
33. Safran M, Ryu J (2014) Ischiofemoral impingement of the hip: a novel approach to treatment. Knee Surg Sport Traumatol Arthrosc 22(4):781-785

34. Sayson SC, Ducey JP, Maybrey JB, Wesley RL, Vermilion D (1994) Sciatic entrapment neuropathy associated with an anomalous piriformis muscle. Pain 59(1):149-152

35. Schatteman J, Vanhoenacker FM, Somville J, Verstraete KL (2015) Ischiofemoral impingement due to a solitary exostosis. JBR-BTR 98(1):39-42

36. Torriani M, Souto SCL, Thomas BJ, Ouellette H, Bredella MA (2009) Ischiofemoral impingement syndrome: an entity with hip pain and abnormalities of the quadratus femoris muscle. AJR Am J Roentgenol 193(1):186-190

37. Tosun Ö, Çay N, Bozkurt M, Arslan H (2012) Ischiofemoral impingement in an 11-year-old girl. Diagn Interv Radiol 18(6):571-573

38. Viala P, Vanel D, Larbi A, Cyteval C, Laredo J-D (2012) Bilateral ischiofemoral impingement in a patient with hereditary multiple exostoses. Skelet Radiol 41(12):1637-1640

39. Volokhina Y, Dang D (2013) Using proximal hamstring tendons as a landmark for ultrasound- and CT-guided injections of ischiofemoral impingement. Radiol Case Rep 8(1):789

40. Wilson MD, Keene JS (2016) Treatment of ischiofemoral impingement: results of diagnostic injections and arthroscopic resection of the lesser trochanter. J Hip Preserv Surg 3(2):146-153

41. Yanagishita CMA, Falótico GG, Rosário DAV, Pugina GG, Wever AAN, Takata ET (2012) Ischiofemoral impingement-an etiology of hip pain: case report. Rev Bras Ortop 47(6):780-783 